\title{
THE HIERARCHY OF CONVEXITY AND SOME CLASSIC INEQUALITIES
}

\section{GHEORGHE TOADER}

Abstract. In what follows, a hierarchy of $m$-convexity is considered: we define $m$-starshaped functions, $m$-superadditive functions, Jensen $m$-convex functions, weak Jensen $m$-convex functions, Jensen $m$-superadditive functions, and weak $m$-superadditive functions. Some inclusions between such classes of functions are established. We also analyze the validity of the HermiteHadamard inequality, and of the Chebyshev-Andersson inequality for $m$-convex functions.

Mathematics subject classification (2000): 26A51, 26D15.

Keywords and phrases: Hermite-Hadamard inequality, Chebyshev-Andersson inequality, hierarchy of $m$-convexity.

\section{REFERENCES}

[1] A. M. BRUCKNer, E. Ostrow, Some function classes related to the class of convex functions, Pacific J. Math., 12 (1962), 1203-1215.

[2] S. S. Dragomir, G. Toader, Some inequalities for $m$-convex functions, Studia Univ. Babeş-Bolyai, 38, 1 (1993), 21-28.

[3] L. FejÉR, On Fourier like sequences (Hungarian), Matem. Term. Ertesitö, 204 (1906), 369-390.

[4] M. KlaRIČIĆ BAKUla, J. PEČARIĆ, M. RIBIČIĆ, Companion inequalities to Jensen's inequalities for m-convex and $(\alpha, m)$-convex functions, J. Inequ. Pure Appl. Math., 7, 5 (2006), Article 194.

[5] V. MineşAn, A generalization of the convexity, Itinerant Sem. Funct. Equat. Approx. Conv., ClujNapoca, Romania, 1993.

[6] D. S. Mitrinović, Analytic Inequalities, Springer Verlag, Berlin, 1970.

[7] P. T. Mocanu, I. Şerb, G. Toader, Real star-convex functions, Studia Univ. Babeş-Bolyai, 42, 3 (1997), 65-80.

[8] G. TOADER, On the hierarchy of convexity of functions, Anal. Numér. Théor. Approx., 15, 2 (1986), $167-172$.

[9] G. TOADER, On a generalization of the convexity, Mathematica, 30, 53 (1988), $183-87$.

[10] G. Toader, On Chebyshev's inequality for functionals, Acta Techn. Napoc., Appl. Math. Mech., 35 (1992), 77-80.

[11] G. TOADER, Superadditivity and Hermite-Hadamard's inequality, Studia Univ. Babeş-Bolyai, 39, 2 (1994), 27-32.

[12] G. ToAder, S. ToAder, Chebyshev-Andersson's inequality, Ineq. Th. Appl., Y. J. Cho, J. K. Kim, S. S. Dragomir, eds., Vol. 3 (2003), 181-188. 